\title{
Lessons from Cancer Immunoediting in Cutaneous Melanoma
}

\author{
Mariana Aris, ${ }^{1,2}$ María Marcela Barrio, ${ }^{2}$ and José Mordoh ${ }^{1,2}$ \\ ${ }^{1}$ Laboratorio de Cancerología, Fundación Instituto Leloir, IIBBA-CONICET, Avenida Patricias Argentinas 435, \\ 1405 Buenos Aires, Argentina \\ ${ }^{2}$ Centro de Investigaciones Oncológicas, Fundación Cáncer and Instituto Alexander Fleming, 1426 Buenos Aires, Argentina \\ Correspondence should be addressed to José Mordoh, jmordoh@leloir.org.ar
}

Received 23 February 2012; Accepted 28 April 2012

Academic Editor: Masoud H. Manjili

Copyright () 2012 Mariana Aris et al. This is an open access article distributed under the Creative Commons Attribution License, which permits unrestricted use, distribution, and reproduction in any medium, provided the original work is properly cited.

\begin{abstract}
We will revisit the dual role of the immune system in controlling and enabling tumor progression, known as cancer immunoediting. We will go through the different phases of this phenomenon, exposing the most relevant evidences obtained from experimental models and human clinical data, with special focus on Cutaneous Melanoma, an immunogenic tumor per excellence. We will describe the different immunotherapeutic strategies employed and consider current models accounting for tumor heterogeneity. And finally, we will propose a rational discussion of the progress made and the future challenges in the therapeutics of Cutaneous Melanoma, taking into consideration that tumor evolution is the resulting from a continuous feedback between tumor cells and their environment, and that different combinatorial therapeutic approaches can be implemented according to the tumor stage.
\end{abstract}

\section{Introduction}

Tumor transformation and progression depends on the cell type and its genetic and epigenetic modifications, where cells overpass several intrinsic tumor suppressor mechanisms and acquire distinctive and complementary capabilities allowing tumor growth and metastatic dissemination [1]. Also, it relies on the interaction of tumor cells with the surrounding environment, the stroma, and the overcoming of extrinsic tumor suppressor mechanisms. In this paper, we will focus on the complex interaction between cancer cells and the immune system, with both controlling and enabling functions, namely, the cancer immunoediting theory. In particular, we will discuss the case of Cutaneous Melanoma (CM), a prototypic immunogenic tumor, and include a critical overview of the different immunotherapeutic approaches employed so far.

\section{Historical Perspective of the Cancer Immunoediting Theory}

The idea that the immune system (IS) is involved in controlling tumor development and progression has been the subject of discussion for many years. In the XX century,
Paul Ehrlich stated the theory of cancer immunosurveillance, reformulated in 1957 by Burnet and Thomas, which proposed that the IS is responsible for preventing tumor development in immunocompetent organisms [2]. They reasoned that cancer would be much more frequent in longlived organisms if it were not for the action of the IS.

The role of IS in tumor control remained controversial until the development of improved genetically-modified murine models of immunodeficiency in the 1990s. Previously, the use of athymic nude mice has mistaken this concept, because no differences were found in tumor incidence between nude and immunocompetent wild type mice [3]. Nowadays, we know that nude mice are not fully immunodeficient, as they have NK cells and some extrathymic Tcell populations [4]. The first supporting evidence proceeded from a landmark work from Robert Schreiber's group, in which the role of IFN- $\gamma$ in tumor surveillance was proven by demonstrating an increased incidence of chemically induced or spontaneously arising tumors in genetically-modified mice deficient for IFN- $\gamma$ or all IFN receptors (Rc) (Stat1-deficient mice), with respect to immunocompetent wild type mice [5]. Similar results were obtained for perforin in a model of spontaneous lymphoma, standing out the relevance of lymphocyte cytotoxicity (NK, NKT, and CD8 cells) in 
preventing tumor development [6]. Later on, the role of NK and NKT cells in protection against carcinogenesis was shown in different experimental models [7]. It was getting clear that mice that lacked components of the innate or the adaptive IS would have a dramatically increased rate of tumor formation. In this regard, additional experiments were performed, revealing that immunodeficient mice were more susceptible to carcinogens than immunocompetent mice [8]. Moreover, it was evidenced that the IS not only controls the number of tumor cells but also their immunogenicity, as tumors developed in immunodeficient mice were more immunogenic (unedited) than similar tumors developed in immunocompetent mice (edited). Therefore, the IS would be involved both in tumor development and in tumor edition of immunogenicity [8]. This stands for the theory of cancer immunoediting, where the IS has a dual role, both suppressing and enabling cancer. It can repress tumor growth by killing cancer cells or arresting proliferation, but it can also enable tumor growth, either by the selection of less immunogenic cells better adapted to survive in an immunocompetent host, or by the establishment of a tumorpermissive microenvironment that enables tumor growth.

\section{The Mains of Cancer Immunoediting}

Several experiments were performed in different immunodeficient murine models, where spontaneous as well as carcinogen-induced tumor development were analyzed, along with the study of immunodeficiency's effect on genetically engineered murine tumor models, which all support and contribute to describe the cancer immunoediting process (reviewed in detail in [9]). The cancer immunoediting theory postulates 3 phases that describe tumor evolution in light of its interaction with the IS: elimination, equilibrium, and escape [2]. Cancer cells communicate with stromal cells either by direct contact or by cytokine and chemokine signaling, proceeding in autocrine and paracrine ways to control and shape tumor growth. And it is the integration of all these signals along with the activation state of the different cell types in the tumor environment that determines whether the equilibrium is displaced to an antitumor response, or to a tumor-permissive environment.

3.1. Elimination. This is the immunosurveillance phase, in which both innate and adaptive immunity work together to detect and destroy tumor cells. This process is an extrinsic tumor suppressor mechanism that acts on cancer cells, in which intrinsic tumor suppressor mechanisms have already failed. In the beginning of tumor development, dying tumor cells and damaged-surrounding tissues release factors like IFN- $\gamma$, IFN $-\alpha / \beta$, and DAMPs [10-12]. These signals recruit cells from innate (NK, NKT, $\gamma \delta$ T cells, macrophages, and dendritic cells) and adaptive IS (CD4 and CD8 T cells). Tumor cells expressing NKG2D activate NK cells. Tumor infiltrating NK cells and macrophages activate each other by production of IFN- $\gamma$ and IL-12, and kill tumor cells by apoptosis via TRAIL, perforins and reactive oxygen and nitrogen species. The activation of dendritic cells promotes the induction of an adaptive immune response, through tumor antigen (Ag) presentation to CD8 cytotoxic T cells (CTL) with help from CD4 cells, ideally generating a long-lasting immune response. Tumor Ag were first evidenced thanks to the finding that mice immunized with carcinogen-induced tumors were protected in case of a new challenge with the same tumor [13]. There are different types of tumor $A g$, including those coded by aberrantly expressed normal genes (melanocyte differentiation $\mathrm{Ag}$ in $\mathrm{CM}$ ); tumor-mutated genes (p53); cancer-testis genes, that in physiological conditions are only expressed in germ cells (MAGE and NY-ESO1 ); and genes encoding viral proteins (HPV proteins).

If the tumor is completely destroyed by the IS, the elimination phase would complete cancer immunoediting. It is important for early tumor control its origin (spontaneous or induced by a carcinogen) as well as its anatomic localization and growth rate. Nowadays, we know that the IS prevents cancer development by different ways: it protects the host from viral infections; it prevents an inflammatory environment that enables tumorigenesis by abruptly removing pathogens; and it eliminates tumor cells by effector cells from the innate and adaptive IS.

In a recent controversial work where, oppositely to the traditional model of primary tumor progression to metastasis, it was proposed that tumor dissemination to secondary organs would be an early event upon transformation, but cancer cells would remain in a dormant state, resulting in staggered metastatic outgrowth [14]. In a murine model of spontaneous melanoma, tumor cells were found to disseminate early in the development of the primary tumor and remain dormant according to the tissue. Dormant cells from lung showed low proliferation rate in comparison to primary tumors, which was partly mediated by cytostatic $\mathrm{CD}^{+} \mathrm{T}$ cells. Therefore, immune strategies that favor the dormancy of disseminated cells can control the development of metastases.

3.2. Equilibrium. This phase takes place when a group of tumor cells survive the initial attack from the IS and move into an equilibrium phase, in which tumor cells are controlled by the IS but cannot be completely eliminated. In this way, tumors can be controlled by the IS for long periods of time, encompassing the host's entire life. T cells, IL-12, and IFN- $\gamma$ are known to sustain the dormancy state [15].

Tumor cells may remain quiescent, with no cell division or apoptosis [16]; or may proliferate and become balanced by apoptosis, with no increase in number [17]. Continuous interaction of the tumor with the IS may lead to the edition of tumor immunogenicity, where cancer cells are modified, generating less immunogenic tumor variants that may escape control by the IS, proliferating and developing clinically detectable tumors.

An experimental model of equilibrium was established by administrating low doses of carcinogen MCA (3-methylcholanthrene) in wild type mice, which was interrupted when tumors arose after CD4, CD8, and IFN $-\gamma$ depletion [15]. However, the same experiment performed in immunodeficient $\mathrm{Rag}^{-/-}$mice did not introduce any change, meaning that disruption of equilibrium would not occur as a result of prolonged de novo transformation. Careful examination 
of the stable mass at equilibrium revealed the presence of atypical cells, with low proliferation index, that induced tumor formation when transplanted into immunodeficient mice. And edited cells from arising tumors were found to be less immunogenic than unedited cells from equilibrium. These experiments showed that cancer cells in equilibrium proliferate poorly and remain unedited, until they spontaneously become edited and escape immune control and grow.

3.3. Escape. Continuous pressure by the IS on genetically unstable cells can lead to the generation of tumor variants that (i) are no longer recognized by the IS, (ii) become insensitive to effector mechanisms, and (iii) induce an immunosuppressor, tolerant microenvironment. Also, changes in the IS are induced that might contribute to a tumorpermissive environment. As a consequence, tumors progress. In Table 1, several of the mechanisms involved in tumor escape are described, including changes in tumor cells and in IS cells, interfering especially with innate and cellular immune response. Supporting references are provided both by experimental models and clinical data from human patients. We will focus on $\mathrm{CM}$, a prototype immunogenic tumor.

\section{Cutaneous Melanoma: A Test Field for Immunotherapy}

$\mathrm{CM}$ is the neoplasia originated from melanocytes that develops in the skin, and it has the fastest growing incidence worldwide [18]. At the clinical-histological level, the Clark model proposes a gradual transition from normal melanocytes to dysplastic nevi, then to primary CM, including radial and vertical growth phases, eventually leading to metastasis (mts) to the lymph nodes (LN) and distant organs [19]. Early diagnosed tumors (stages I-II, AJCC) are curable by surgery in more than $90 \%$ of cases; however, when CM metastasizes, only a minority of patients can be cured [20]. CM would not respond to conventional therapies like radiotherapy and chemotherapy; nevertheless, as it is an immunogenic tumor, it allows the use of immunotherapy as an alternative. Among the strongest supporting evidences for the dual role of the IS in CM eradication and progression are included the following:

(a) Tumor Antigens. The presence of tumor Ag in humans was shown by modern methodologies, involving the use of antibodies and CTL derived from patients as probes, tested on autologous tumor cell libraries. Among CM Ag outstands melanocyte differentiation Ag (MD-Ag) such as MART-1 [21, 22], gp100 [23], tyrosinase [24], tyrosinaserelated protein-1 (TRP1) [25], TRP2 [26], and MELOE-1 [27]; cancer-testis Ag from the MAGE super-family [28] and NY-ESO-1 [29]; and tumor-mutated Ag such as BRAF [30].

(b) Spontaneous regressions. The finding of both humoral and cellular immunity to tumor Ag suggests that the IS is capable of eliciting a coordinated immune response to tumors as it would to a foreign Ag. Indeed, several tumor regressions were observed in patients as a consequence of the action of the IS [31]. Infiltration of IS cells is an early event in transformation and it is associated with disease outcome. There are several studies in CM patients that correlate quantity, quality, and distribution of tumor infiltrating lymphocytes (TIL) with patient survival [19, 32, 33]. The first studies just analyzed the presence and distribution of lymphocytes; more recent studies also focus on the immunophenotype of IS cells, as it is known that the IS may move from an antitumor environment to a tumorpermissive one. It was described in a case report a CM patient treated with anti-CTLA-4 therapy that was undergoing simultaneously the three phases of cancer immunoediting, with regressing, stable, and progressing lesions [34]. Probably, the environment of the different metastases (mts) would account for tumor response. In another clinical case, different rounds of immunoediting, escape and immune adaptation by shifting of the T-cell response were observed [35].

(c) Immunodeficiency. In general, immunodeficiency is associated with an increased risk of developing cancer. Most related factors include viral oncogenesis and reduced tumor immunosurveillance. Immunocompromised patients, like transplant recipients or AIDS patients, develop lymphomas (Epstein-Barr virus), Kaposi's sarcoma (Herpes virus), and cervical cancer (Human papillomavirus) [36]. An increased incidence of tumors non-related to virus, like colon, lung, pancreas, kidney, head and neck, skin carcinomas and CM was also observed [37]. In a case report, it was described that two patients that received kidney grafts from a common donor developed CM. It was further revealed that the donor had overcome this pathology in the past; therefore, the donor's kidney probably contained CM cells held in equilibrium by the IS. When kidneys were grafted into immunosuppressed recipients, the development of CM was favored [38]. These evidences are consistent with the idea that tumors progress in immunosuppressive permissive environments.

(d) Immunosuppression. Although CM is highly immunogenic, tumors develop and progress in immunocompetent patients. One of the contributing factors is the induction of a local state of immune suppression and tolerance to tumors as a result of tumor interaction with its environment. Cancer cells develop different mechanisms for tumor escape, including evasion of $\mathrm{Ag}$ recognition by the IS and secretion of immunosuppressor and proapoptotic factors (Table 1). Analysis of immunosuppressor factors in primary CM biopsies, negative and positive sentinel lymph nodes (SLN), and $\mathrm{LN}$ with advanced metastasis revealed that primary $\mathrm{CM}$ cells secreted TGF- $\beta 2$ that renders dendritic cells tolerogenic; tolerogenic dendritic cells (tDC) and Treg were found at all stages, with increasing IDO and IL-10 secretion with CM progression, making the SLN an immunoprivileged site suitable for metastasis [39]. Thus, tumor cells would secrete immunosuppressor factors that would render IS effector cells into a tolerant phenotype, which in turn would secrete more 


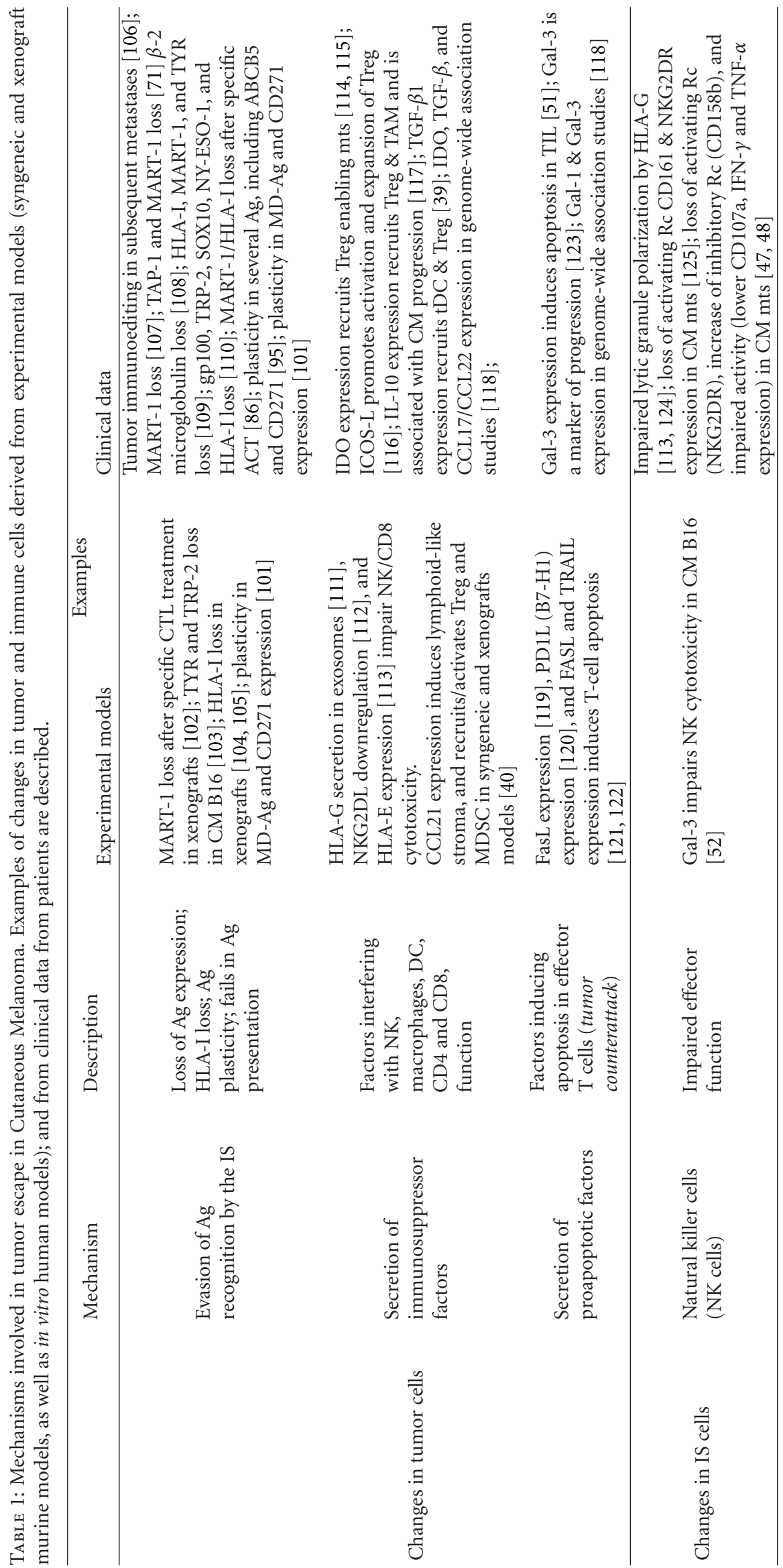




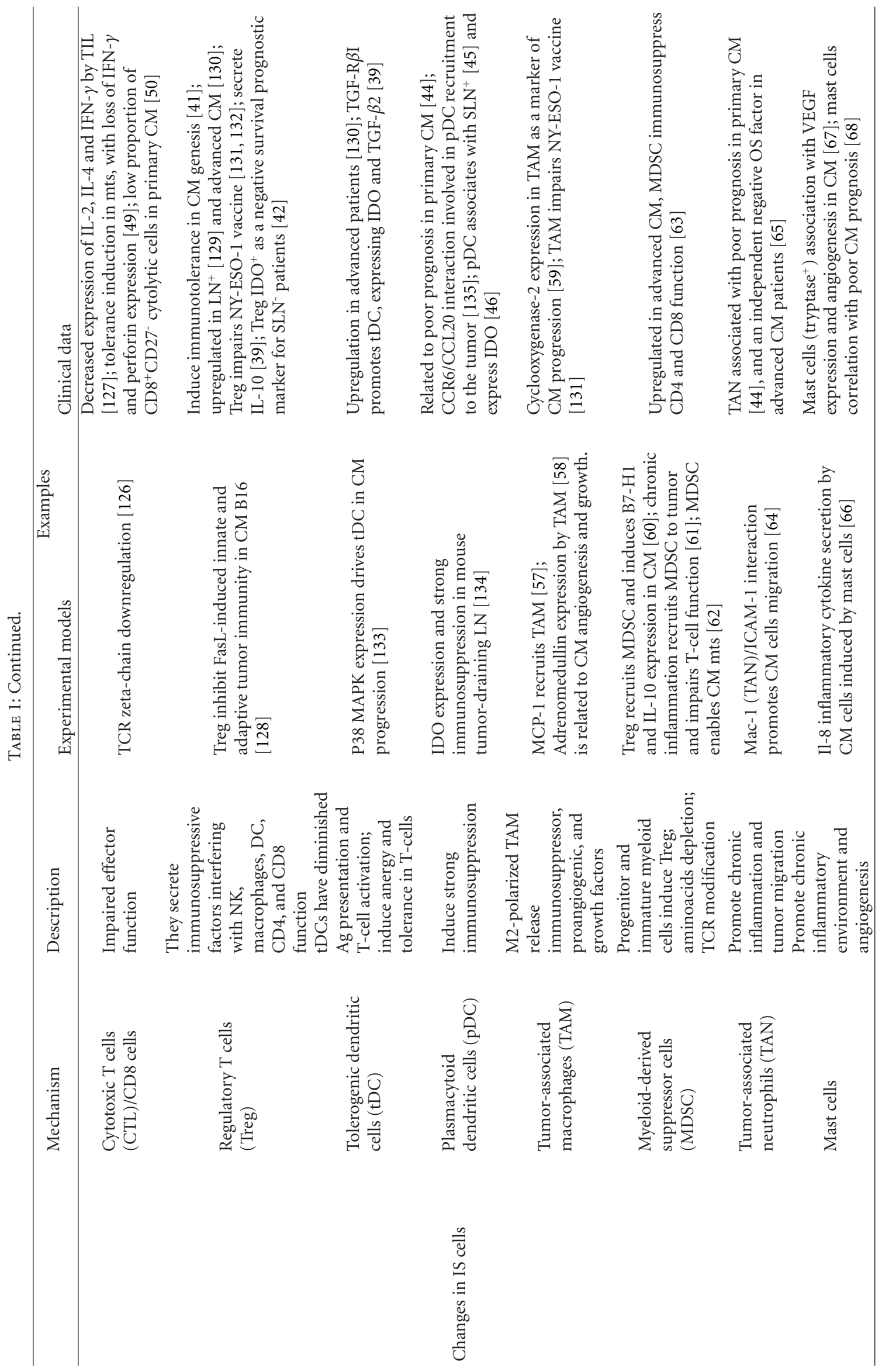


immunosuppressor factors preparing the niche for metastasis before tumor dissemination. Recently, it was shown that secretion of CCL21 by melanoma cells promotes tolerance in syngeneic and xenograft CM models [40]. CCL21 $1^{\text {low }}$ tumors presented specific CTL for CM Ag and cytokines related to an immunogenic response. Instead, CCL2 $1^{\text {high }}$ tumors secrete TGF- $\beta 1$, promote CCR7-dependent Treg and MDSC activation, and increased lymphoid tissue inducer cells, which promoted lymphoid neogenesis.

Regulatory $T$ cells (Treg) are key immunosuppressor factors. The presence of $\mathrm{CD} 4^{+} \mathrm{CD} 25^{+} \mathrm{FOXP}^{+}$Treg was analyzed among different nevi (common/atypical junctional and compound nevi, Spitz nevi) and primary CM [41]. These regulatory cells were found in all these lesions, but were more represented in atypical junctional/compound nevi and in radial growth phase CM, suggesting that Treg induce immunotolerance early during CM genesis, favoring $\mathrm{CM}$ growth. Indeed, Indoleamine 2,3-dioxygenase (IDO) expression in Treg, an enzyme with immunosuppressive properties, was identified as a negative survival prognostic marker in $\mathrm{SLN}^{-}$patients [42]. Moreover, tumor Ag-specific $\mathrm{CD}^{+} \mathrm{CD}^{2} 5^{+} \mathrm{FoxP}^{+}$Treg were evidenced in the blood of patients with metastatic CM [43]. These cells recognized a broad range of tumor Ag, including gp100, NY-ESO-1, TRP1 and inhibitor of apoptosis protein (IAP), and proliferated in response to specific peptides. They produced preferentially IL-10 and suppressed autologous $\mathrm{CD} 4^{+} \mathrm{CD} 25^{-}$T-cell responses in a cell contact-dependent manner; they were not detected in healthy individuals. Therefore, these tumorAg-specific Treg might represent a target for improving CM immunotherapy.

Plasmacytoid dendritic cells (pDC) are characterized by the induction of strong immunosuppression. Both $\mathrm{pDC}$ and neutrophils were found associated with pSTAT-3 expression in $\mathrm{CM}$, resulting in markers of poor prognosis in primary $\mathrm{CM}$ [44]. Also, pDC accumulate in $\mathrm{SLN}^{+}$and express IDO, promoting immunotolerance $[45,46]$.

With regard to effector cells, a natural function of NK lymphocytes is to kill cells that fail to express MHC I molecules, thereby contributing to tumor eradication. The most frequent event observed in NK cells during CM progression is loss of activating Rc and increase of inhibitory Rc [47, 48]. With respect to CTL, the induction of an immunotolerant state interferes with the cytotoxic function of CTLs, as IFN$\gamma$ and perforin expression decrease $[49,50]$. Also, tumor cells secrete factors, like Galectin-3, that induce apoptosis of CTL and NK cells $[51,52]$. With regard to tolerance, the functional state of tumor-specific CTLs anti-MART-1 from peripheral blood and metastasis populations from CM patients was compared [49]. TILs expressed lower levels of IFN- $\gamma$ and perforin than peripheral $\mathrm{T}$ cells, indicating a local state of tolerance. However, cytotoxic activity could be recovered after re-stimulation of CD8 cells by in vitro culture; therefore, local induction of tolerance would be reversible.

(e) Inflammation. Chronic inflammation is a key factor involved in tumor development and progression (reviewed in [53]). Sun exposure promotes an inflammatory environment in the skin, increasing the risk of developing skin cancer, including CM [54]. Inflammation contributes to tumor initiation by increasing the DNA mutation rate, and through production of reactive oxygen and nitrogen species that induce DNA damage and instability. Also, it activates tissue repair responses, inducing proliferation of premalignant cells and enhancing their survival. Tumor-infiltrating IS cells secrete cytokines that activate key transcription factors in transformed cells, like NF $\kappa$ B or STAT-3, that control survival, proliferation, growth, angiogenesis, and invasion [55]. In turn, these transcription factors induce chemokines that attract additional inflammatory IS cells to sustain tumorassociated inflammation. Upon transformation, inflammation stimulates angiogenesis and causes local immunosuppression, helping tumor cells to survive and accumulate additional mutations as well as epigenetic changes, enabling tumor progression.

Macrophages are key mediators of the inflammatory response. Macrophages can be classified into M1 and M2 types [56]. M1 macrophages are associated with the acute inflammatory response, capable of killing pathogens, and priming antitumor immune responses. They can be activated by IFN- $\gamma$ and pathogens and express high levels of proinflammatory cytokines (TNF- $\alpha$, IL-1, IL-6, IL-12, or IL23), MHC molecules and NO (nitric oxide) synthase. On the other hand, M2 macrophages (or "alternatively" activated macrophages), induced in vitro by IL-4, IL-10, and IL-13, downregulate MHCII and IL-12 expression and increase IL10 , scavenger receptor $\mathrm{A}$, and arginase. This phenotype is related to an inflammatory tumor-permissive environment. However, M1 and M2 macrophages phenotype is plastic since it is defined by gene expression profiles; oppositely to CD4 $\mathrm{T}_{H} 1$ and $\mathrm{T}_{H} 2$ cells, which involved differentiation committed pathways. In CM, M2 tumor-associated macrophages (TAM) release tumor-enabling factors, including angiogenic and growth factors. Overexpression of monocyte chemoattractant protein (MCP-1) on CM cells attracted macrophages, enabling tumor growth and angiogenesis in a human xenograft model [57]. Also, adrenomedullin expression by TAM enables tumor growth and angiogenesis in CM B16 [58]. Analysis of TAM at different stages including benign nevi revealed more frequency of $\mathrm{COX}-2^{+}$TAM in primary $\mathrm{CM}$, proposing $\mathrm{COX}-2$ as a marker of $\mathrm{CM}$ progression [59].

Myeloid-derived suppressor cells (MDSC), a heterogeneous group of progenitor and immature myeloid cells, have emerged as key immune modulators in various human malignancies. In several experimental models, it was shown that chronic inflammation recruits MDSC to the tumor, expressing immunosuppressor factors, impairing T-cell function and enabling metastases [60-62]. MDSC frequency is increased with CM progression, and STAT-3 is a key factor in MDSC development and function [63].

Tumor-associated neutrophils (TAN) were shown to promote CM cells migration via MAC-1/ICAM-1 interaction [64], and to be associated with poor prognosis at all stages $[44,65]$. Finally, mast cells are also implied in tumorassociated inflammation. TNF- $\alpha$ and histamine secreted by mast cells induced expression of the inflammatory cytokine 
IL-8 by CM cells [66]. Tryptase ${ }^{+}$mast cells were found related to VEGF expression, enabling angiogenesis in CM [67]; therefore, they were associated with poor prognosis for CM [68].

On this basis, different therapeutic approaches were employed with the main purposes of overcoming chronic inflammation, immunosuppression, and tolerance induced by the own tumor and its environment, and to stimulate tumor Ag immunogenicity and effector function of immune cells in order to eradicate tumors. We will discuss different examples, highlighting evidences from cancer immunoediting, and progresses and challenges in the treatment of CM.

4.1. Adjuvant Therapies. Among drugs used to stimulate immune effector cells, high-dose Interferon alfa2B (IFN- $\alpha 2 \mathrm{~b}$ ) is a FDA-approved drug for use in patients with stages IIIII CM. Whereas it increases disease-free survival and has a moderate effect on overall survival (OS), it is nevertheless related to severe side effects [69]. Another adjuvant therapy commonly employed, interleukin-2 (IL-2), promotes proliferation of T, B, and NK cells. This drug is approved for stage IV CM patients, with $16 \%$ objective response (OR) rate, including $6 \%$ complete response rate, although associated with short-term acute toxicity [70]. In a case report, a patient presented loss of the TAP-1 and MD-Ag MART-1 within subsequent metastases developed after several therapies, including IL-2 [71]. Cytogenetic analysis of the subsequent metastases revealed similar profiles, indicating a common genetic composition. Sensitivity to previous CTL clones could be restored by introducing MART- 1 and TAP- 1 by retroviral expression, further supporting the immunoediting of this tumor during its progression.

4.2. Molecular Target-Specific Therapies. Among targetspecific therapies are the blockade of oncogenes. The most frequent mutation found in CM (50-65\%) is a driver mutation in the BRAF oncogene, $\mathrm{BRAF}^{\mathrm{V} 600 \mathrm{E}}$, involved in the MAPK proliferation pathway [30]. Specific inhibitors were designed for $\mathrm{BRAF}^{\mathrm{V} 600 \mathrm{E}}$ and tested on advanced $\mathrm{CM}$ patients. In a phase III study, comparison of the $\mathrm{BRAF}^{\mathrm{V} 600 \mathrm{E}}$ inhibitor Vemurafenib with the chemotherapeutic drug Dacarbazine showed more than $50 \%$ of response rate to Vemurafenib, with a sensitive increase in overall survival and progressionfree survival (PFS) in comparison to Dacarbazine [72]. This inhibitor allowed achieving important remissions, although transitory, because relapses were observed. However, the administration of Vemurafenib does not interfere neither with the viability nor functionality of $\mathrm{T}$ cells, allowing the implementation of a combinatorial approach with immunotherapy [73]. The increase in the flow of CD4 and CD8 cells to the tumor site after beginning of Vemurafenib administration further supports combinatorial strategies [74]. In a recent landmark work, in $\mathrm{MYC}^{+}$and BCR$\mathrm{ABL}^{+}$lymphoma and leukemia mouse models of oncogene addiction, it was shown that $\mathrm{CD} 4^{+}$cells are involved in cellular senescence, shutdown of angiogenesis and chemokine expression [75]. This provides evidence that the IS plays a role in tumor regression upon oncogene inactivation, a process that was considered cell-autonomous, adding scientific rationale for combination therapeutic approaches.

4.3. Immune Tumor-Specific Therapies. The main routes employed to promote tumor-specific immunity include the active way through the use of therapeutic vaccines (in vivo), and the passive way through Adoptive Cell Therapy (ACT) (ex vivo). Therapeutic vaccines are administered after surgery with the purpose of mounting a long-lasting immunity and controlling any micrometastatic foci. The rational base of vaccines is that tumor Ag must be captured by dendritic cells, which migrate to lymph nodes to activate CD4 and CD8 cells, triggering an adaptive immune response. Therapeutic vaccines include tumor $\mathrm{Ag}$ vaccines, with different sources of Ag, like peptides, tumor lysates, recombinant virus, or whole irradiated cells; dendritic cells vaccines, consisting of autologous dendritic cells stimulated in vitro with a proper Ag source, maturated ex vivo, and then injected back into the patient. ACT involves the expansion of autologous T cells ex vivo to achieve sufficient number to eliminate important tumor masses. Either TILs or genetically modified T cells (with clonotypic TCR or chimeric antigenic receptor) might be perfused. This is an attractive approach for advanced patients whose tumors cannot be removed by surgery. However, the convenience of this expensive treatment has not yet been validated in randomized, prospective clinical trials.

With regard to tumor Ag vaccines, in a trial with MART1, gp100 and tyrosinase peptides in metastatic CM patients, one of the patients who experienced a dramatic tumor regression had preexisting immunity to TRP2 and NYESO-1 Ag [76]. Of interest, the immune reactivity against TRP2 persisted over time, whereas that against NY-ESO1 waned over the course of follow up; it is possible that residual tumors were immunoselected in vivo for loss of NYESO-1 over time. Other clinical cases of tumor regression upon vaccination with MAGE-1 peptide indicated a higher frequency of CTL towards general tumor Ag (antitumor CTL) than specific vaccine-Ag (anti-vaccine CTL), both in the tumor and in circulation $[77,78]$. Antitumor T cells were already present in the patient before vaccination, with some highly dominant clonotypes. Thus, preexisting antitumor $\mathrm{T}$ cells may be ineffective at rejecting the tumor either because their frequency is too low, because tumor cells were selected to escape recognition, or because such lymphocytes are functionally deficient. However, this state of functional tolerance might be reversed by the administration of vaccines. A possible explanation is that vaccination induces cytokine cascades both locally and systemically, resulting in activation and proliferation of anti-melanoma Ag precursors, and infiltration of these effectors into tumors. Thus, a spontaneous antitumor T-cell response, which has become ineffective, can be reversed by vaccination and contribute to tumor rejection.

Actually, vaccination with tumor-Ag vaccines has been extensively assayed in CM patients, so far with little success $[79,80]$. What could be the reasons for the failure of therapeutic vaccination in a large majority of the patients? A possible explanation is the low occurrence of anti-vaccine $\mathrm{T}$ cells that have the required functional properties to migrate to the tumor, resist the inhibitory tumor environment, and 
initiate focal activation. Another factor might be the inability to overcome the severe immunosuppressive environment, preventing the effectiveness of any vaccine. In fact, recent studies indicate that selective Treg depletion improves therapeutic effect of vaccines [81]. Another issue is that vaccines that target a unique Ag are in disadvantage, since tumors in general are heterogeneous, CM being not an exception. Therefore resistance may come from the coexistence of heterogeneous populations, or acquired by loss of $\mathrm{Ag}$ or HLA expression. In this regard, targeting multiple targets provides a step forward. In this way, previous clinical trials conducted by us indicated that vaccination with allogeneic irradiated cells, in patients in early stages of the disease, may prolong significantly disease-free survival [82]. Also, promising results were obtained for patients with stages II/III CM in a phase I study with autologous dendritic cells loaded ex vivo with allogeneic irradiated cells $[83,84]$.

With regard to ACT, there are also different strategies. Culture of TIL is not suitable for all patients because of technical issues (reviewed in [85]), but achieved 50\% OR in different trials $[86,87]$. This procedure requires a previous cycle of immunosuppression in order to suppress the endogenous immunosuppressive environment from patients. This OR increased to $72 \%$ with more severe immunosuppressive before treatment, but required hematopoietic stem cells transfusion afterwards [88]. Young TIL protocols introduced shorter culture times, although administered TILs were unselected; however, it achieved $50 \%$ OR in a phase II study [89]. A limitation of this approach is the requirement that patients have preexisting tumor reactive cells that can be expanded ex vivo. Genetically modified $\mathrm{T}$ cells are derived from patient's blood cells, therefore are more feasible to be obtained. Cells transduced with most frequent tumorregression Ag, MART-1 and gp100, allowed to achieve for MART-1 12\% OR [90]; and 30\% and 19\% OR for MART-1 and gp100-specific CTL in another phase I study, including remissions of brain metastases [91]. Studies with cells modified with chimeric antigenic receptors for CM therapy are on the way. The principal disadvantage of specific CTLs/genetically modified T cells is that they target a unique $\mathrm{Ag}$, easing the development of resistance. In a phase I study of TIL immunotherapy, although half of the patients presented clinical responses, almost $60 \%$ showed evidence of immunoediting with loss of MART-1 or HLA-I [86]. In other phase-I studies, the effect of MART-1 specific CTL clones in advanced-stage patients was analyzed, with half of the responders showing loss of $\mathrm{Ag}$ expression $[92,93]$.

\section{Cutaneous Melanoma Heterogeneity and Immune Response}

One question that arises from the observation of limited clinical responses and remissions in Ag-targeted therapies is about the nature of tumor growth and heterogeneity observed in CM. Whether there are different proliferative populations hierarchically organized, with distinguishing Ag, or there are unstable populations with variable proliferative potential. The cancer stem cell (CSC) model proposes a cellular hierarchy within tumors in which, as in physiological tissues, only the minor CSC subset would have unlimited proliferative potential, being capable of self-renewal and generation of differentiated cells, accounting for the tumor mass [94]. Oppositely, the stochastic or clonal evolution model states that most cells would self-renew, accounting for tumor growth. A more dynamic model of phenotypic plasticity is gaining momentum, in which cells would have a proliferative potential variable in time [95]. This is an important issue, since recent publications described that $\mathrm{CM}$ CSC, selected by CD271 expression, would not express MDAg MART-1, gp100, and tyrosinase [96]. Also, CM CSC selected by ABCB5, would not express MART-1 [97]. In contrast, it was reported that one out of four CM cells developed tumors in NOD/SCID Il2 $\mathrm{rg}^{-/-}$mice without any previous selection [98]; and that phenotypic plasticity, even in CSC markers like CD271 or ABCB5, would be a source of heterogeneity in CM [95].

We were interested in the study of the expression of immunotherapy-relevant $\mathrm{Ag}$ in $\mathrm{CM}$ proliferative populations. In particular, we wanted to disclose if cells expressing MD-Ag have limited proliferative potential, thus allowing MD-Ag non-expressing clonogenic cells (CC) to survive immune effectors and repopulate the tumor; we also wanted to address if CC would be intrinsically resistant to CTL. We focused on MART-1 and gp100, since in HLA-A0201 patients (40\% Latin-Americans), most TILs are directed against them, thus appearing to be the most frequent Ag involved in tumor regressions $[99,100]$. We analyzed MART-1/gp100 and the proliferation marker Ki-67 expression in primary and metastatic CM biopsies, observing the coexistence of MART-1/gp100 expressing and non-expressing populations that proliferated competitively, with no differences between primary and metastatic tumors. However, cells with differential proliferative potential might replicate. Therefore, we analyzed MART-1, gp100, tyrosinase, and CD271 expression in colonies obtained from anchorage-independent growing CC of human CM cell lines. By 7 days, colonies displayed positive, negative, and mixed expression patterns. By 14 days, Ag were downregulated, suggesting Ag plasticity. We found that plasticity in MART-1 expression involves promoter methylation. We studied MART-1 and gp100 expression along time in $\mathrm{CM}$ growing clones, revealing that Ag levels varied with time without interfering with clonogenicity. Finally, CC MART-1/gp100 expressing cells were efficiently lysed by specific CTL. In conclusion, we found that MD-Ag or CSC marker CD271 expression would not interfere neither with proliferation nor clonogenicity, and CC expressing the proper Ag and HLA-class-I haplotype would not be intrinsically resistant to lysis by CTL. Since MD-Ag-expressing and non-expressing cells are proliferative and clonogenic, giving rise to colonies of thousand cells, both subpopulations should be considered as targets to eradicate tumors [101].

\section{Conclusions}

What do we know about cancer immunoediting? Extensive research in this field reveals that there is a continuous feedback between the tumor and its microenvironment that 
determines tumor fate. It is not a fixed interaction, but rather a dynamic one, where signals from cancer and surrounding cells are constantly modifying each other giving an integral response. However, tumor evolution is progressive, and its study provides the possibility to interfere with this process with different therapeutic approaches according to the tumor stage (different stages; different approaches).

What have we learned about CM and immunotherapy? $\mathrm{CM}$ is a prototypic immunogenic tumor, with spontaneous regressions described in patients and with several Ag identified. Due to its limited therapeutic options when it metastasizes, immunotherapy has emerged as a remarkable one. In clinical practice, Ag-targeted therapies, even with vaccines or CTL, have achieved modest success. Among contributing factors, the specific blockade of the tumor immunosuppressor environment is a high wall to climb but it is indeed necessary, preserving as much as possible the immune repertoire from the patient. Also, it is relevant to overcome chronic inflammation, which fosters genomic instability, immunosuppression, growth, and angiogenesis in tumors. With regard to tumor cell heterogeneity, it is important to discern whether Ag heterogeneity is due to the presence of differentiated cells with limited proliferative potential (CSC model), or to Ag plasticity independent from proliferation (phenotypic plasticity model), accounting for resistance and escape from immune effectors. We and others found $\mathrm{Ag}$ and phenotypic plasticity even in CSC markers. Thus, if Ag expression varies in time, immunotherapeutic approaches should point towards plasticity or multitargeting of $\mathrm{Ag}$, so at least some responder $\mathrm{T}$-cell clones with proper migration capability and resistance to inhibitory factors would be obtained. In advanced patients, the equilibrium phase has largely been displaced, with a highly tumor immunosuppressive environment, many times with unresectable tumors. However, exciting approaches have arisen from studies of $\mathrm{CM}$ biology, like the use of $\mathrm{BRAF}^{\mathrm{V} 600 \mathrm{E}}$ inhibitors; recently, the role of the IS upon oncogene inactivation was evidenced, providing support for combinatorial therapeutic strategies.

What do we learn about cancer immunoediting for improving therapeutic strategies? We learn that the cancer immunoediting process considers the tumor as an integral organ with different components, including cancer cells as well as stromal cells, and so should therapeutic approaches do. Certainly, the continuous study of CM biology and its environment will improve combinatorial therapeutic approaches to reach an equilibrium state or, best of all, achieve tumor eradication.

\section{Abbreviations}

ACT: Adoptive cell therapy

Ag: Antigen/s

CSC: Cancer stem cells

CC: Clonogenic cells

CM: Cutaneous melanoma

IDO: Indoleamine 2,3-dioxygenase

IS: Immune system

LN: Lymph node

mts: Metastasis/metastases
MDSC: Myeloid-derived suppressor cells

pDC: Plasmacytoid dendritic cells

PFS: Progression-free survival

OR: Objective response

OS: Overall survival

SLN: Sentinel lymph node

Rc: Receptor

TAM: Tumor-associated macrophages

TAN: Tumor-associated neutrophils

tDC: Tolerogenic dendritic cells

TIL: Tumor-infiltrating lymphocytes.

\section{Conflict of Interests}

The authors indicate that they have no potential conflict of interests.

\section{References}

[1] D. Hanahan and R. A. Weinberg, "Hallmarks of cancer: the next generation," Cell, vol. 144, no. 5, pp. 646-674, 2011.

[2] R. D. Schreiber, L. J. Old, and M. J. Smyth, "Cancer immunoediting: integrating immunity's roles in cancer suppression and promotion," Science, vol. 331, no. 6024, pp. 15651570, 2011.

[3] O. Stutman, "Tumor development after 3 methylcholanthrene in immunologically deficient athymic nude mice," Science, vol. 183, no. 4124, pp. 534-536, 1974.

[4] G. P. Dunn, A. T. Bruce, H. Ikeda, L. J. Old, and R. D. Schreiber, "Cancer immunoediting: from immunosurveillance to tumor escape," Nature Immunology, vol. 3, no. 11, pp. 991998, 2002.

[5] D. H. Kaplan, V. Shankaran, A. S. Dighe et al., "Demonstration of an interferon $\gamma$-dependent tumor surveillance system in immunocompetent mice," Proceedings of the National Academy of Sciences of the United States of America, vol. 95, no. 13, pp. 7556-7561, 1998.

[6] M. J. Smyth, K. Y. Thia, S. E. Street, D. MacGregor, D. I. Godfrey, and J. A. Trapani, "Perforin-mediated cytotoxicity is critical for surveillance of spontaneous lymphoma," Journal of Experimental Medicine, vol. 192, no. 5, pp. 755-760, 2000.

[7] M. J. Smyth, K. Y. Thia, S. E. Street et al., "Differential tumor surveillance by natural killer (NK) and NKT cells," Journal of Experimental Medicine, vol. 191, no. 4, pp. 661-668, 2000.

[8] V. Shankaran, H. Ikeda, A. T. Bruce et al., "IFN $\gamma$, and lymphocytes prevent primary tumour development and shape tumour immunogenicity," Nature, vol. 410, no. 6832, pp. 1107-1111, 2001.

[9] M. D. Vesely, M. H. Kershaw, R. D. Schreiber, and M. J. Smyth, "Natural innate and adaptive immunity to cancer," Annual Review of Immunology, vol. 29, pp. 235-271, 2011.

[10] S. E. Street, E. Cretney, and M. J. Smyth, "Perforin and interferon- $\gamma$ activities independently control tumor initiation, growth, and metastasis," Blood, vol. 97, no. 1, pp. 192197, 2001.

[11] G. P. Dunn, A. T. Bruce, K. C. Sheehan et al., "A critical function for type I interferons in cancer immunoediting," Nature Immunology, vol. 6, no. 7, pp. 722-729, 2005.

[12] G. P. Sims, D. C. Rowe, S. T. Rietdijk, R. Herbst, and A. J. Coyle, "HMGB1 and RAGE in inflammation and cancer," Annual Review of Immunology, vol. 28, pp. 367-388, 2010. 
[13] L. J. Old and E. A. Boyse, "Immunology of experimental tumors," Annual Review of Medicine, vol. 15, pp. 167-186, 1964.

[14] J. Eyles, A. L. Puaux, X. Wang et al., “Tumor cells disseminate early, but immunosurveillance limits metastatic outgrowth, in a mouse model of melanoma," The Journal of Clinical Investigation, vol. 120, no. 6, pp. 2030-2039, 2010.

[15] C. M. Koebel, W. Vermi, J. B. Swann et al., "Adaptive immunity maintains occult cancer in an equilibrium state," Nature, vol. 450, no. 7171, pp. 903-907, 2007.

[16] G. N. Naumov, I. C. MacDonald, A. F. Chambers, and A. C. Groom, "Solitary cancer cells as a possible source of tumour dormancy?" Seminars in Cancer Biology, vol. 11, no. 4, pp. 271-276, 2001.

[17] L. Holmgren, M. S. O’Reilly, and J. Folkman, "Dormancy of micrometastases: balanced proliferation and apoptosis in the presence of angiogenesis suppression," Nature Medicine, vol. 1, no. 2, pp. 149-153, 1995.

[18] R. Siegel, E. Ward, O. Brawley, and A. Jemal, "The impact of eliminating socioeconomic and racial disparities on premature cancer deaths," CA-A Cancer Journal for Clinicians, vol. 61, pp. 212-236, 2011.

[19] W. H. Clark Jr., D. E. Elder, Dt Guerry et al., "Model predicting survival in stage I melanoma based on tumor progression," Journal of the National Cancer Institute, vol. 81, no. 24, pp. 1893-1904, 1989.

[20] S. B. Edge and C. C. Compton, "The American Joint Committee on Cancer: the 7th edition of the AJCC cancer staging manual and the future of TNM," Annals of Surgical Oncology, vol. 17, no. 6, pp. 1471-1474, 2010.

[21] P. G. Coulie, V. Brichard, A. Van Pel et al., "A new gene coding for a differentiation antigen recognized by autologous cytolytic T lymphocytes on HLA-A2 melanomas," Journal of Experimental Medicine, vol. 180, no. 1, pp. 35-42, 1994.

[22] Y. Kawakami, S. Eliyahu, C. H. Delgado et al., "Cloning of the gene coding for a shared human melanoma antigen recognized by autologous T cells infiltrating into tumor," Proceedings of the National Academy of Sciences of the United States of America, vol. 91, no. 9, pp. 3515-3519, 1994.

[23] Y. Kawakami, S. Eliyahu, C. H. Delgado et al., "Identification of a human melanoma antigen recognized by tumorinfiltrating lymphocytes associated with in vivo tumor rejection," Proceedings of the National Academy of Sciences of the United States of America, vol. 91, no. 14, pp. 6458-6462, 1994.

[24] V. Brichard, A. Van Pel, T. Wolfel et al., "The tyrosinase gene codes for an antigen recognized by autologous cytolytic $\mathrm{T}$ lymphocytes on HLA-A2 melanomas," Journal of Experimental Medicine, vol. 178, no. 2, pp. 489-495, 1993.

[25] T. Cohan, R. M. Muller, Y. Tomita, and S. Shibahara, "Nucleotide sequence of the cDNA encoding human tyrosinase-related protein," Nucleic Acids Research, vol. 18, no. 9, pp. 2807-2808, 1990.

[26] R. F. Wang, E. Appella, Y. Kawakami, X. Kang, and S. A. Rosenberg, "Identification of TRP-2 as a human tumor antigen recognized by cytotoxic T lymphocytes," Journal of Experimental Medicine, vol. 184, no. 6, pp. 2207-2216, 1996.

[27] Y. Godet, A. Moreau-Aubry, Y. Guilloux et al., "MELOE-1 is a new antigen overexpressed in melanomas and involved in adoptive T cell transfer efficiency," Journal of Experimental Medicine, vol. 205, no. 11, pp. 2673-2682, 2008.

[28] P. van der Bruggen, C. Traversari, P. Chomez et al., "A gene encoding an antigen recognized by cytolytic $\mathrm{T}$ lymphocytes on a human melanoma," Science, vol. 254, no. 5038, pp. 1643-1647, 1991.

[29] Y. T. Chen, M. J. Scanlan, U. Sahin et al., "A testicular antigen aberrantly expressed in human cancers detected by autologous antibody screening," Proceedings of the National Academy of Sciences of the United States of America, vol. 94, no. 5, pp. 1914-1918, 1997.

[30] H. Davies, G. R. Bignell, C. Cox et al., "Mutations of the BRAF gene in human cancer," Nature, vol. 417, no. 6892, pp. 949-954, 2002.

[31] L. V. Kalialis, K. T. Drzewiecki, and H. Klyver, "Spontaneous regression of metastases from melanoma: review of the literature," Melanoma Research, vol. 19, no. 5, pp. 275-282, 2009.

[32] C. G. Clemente, M. C. Mihm Jr., R. Bufalino, S. Zurrida, P. Collini, and N. Cascinelli, "Prognostic value of tumor infiltrating lymphocytes in the vertical growth phase of primary cutaneous melanoma," Cancer, vol. 77, no. 7, pp. 1303-1310, 1996.

[33] I. S. van Houdt, B. J. Sluijter, L. M. Moesbergen et al., "Favorable outcome in clinically stage II melanoma patients is associated with the presence of activated tumor infiltrating Tlymphocytes and preserved MHC class I antigen expression," International Journal of Cancer, vol. 123, no. 3, pp. 609-615, 2008.

[34] J. Yuan, D. B. Page, G. Y. Ku et al., "Correlation of clinical and immunological data in a metastatic melanoma patient with heterogeneous tumor responses to ipilimumab therapy," Cancer Immunity, vol. 10, article 1, 2010.

[35] G. V. Yamshchikov, D. W. Mullins, C. C. Chang et al., "Sequential immune escape and shifting of T cell responses in a long-term survivor of melanoma," Journal of Immunology, vol. 174, no. 11, pp. 6863-6871, 2005.

[36] M. Frisch, R. J. Biggar, E. A. Engels, and J. J. Goedert, "Association of cancer with AIDS-related immunosuppression in adults," Journal of the American Medical Association, vol. 285, no. 13, pp. 1736-1745, 2001.

[37] C. M. Vajdic, S. P. McDonald, M. R. McCredie et al., "Cancer incidence before and after kidney transplantation," Journal of the American Medical Association, vol. 296, no. 23, pp. 28232831, 2006

[38] R. M. MacKie, R. Reid, and B. Junor, "Fatal melanoma transferred in a donated kidney 16 years after melanoma surgery," The New England Journal of Medicine, vol. 348, no. 6, pp. 567568, 2003.

[39] M. E. Polak, N. J. Borthwick, F. G. Gabriel et al., "Mechanisms of local immunosuppression in cutaneous melanoma," British Journal of Cancer, vol. 96, no. 12, pp. 1879-1887, 2007.

[40] J. D. Shields, I. C. Kourtis, A. A. Tomei, J. M. Roberts, and M. A. Swartz, "Induction of lymphoidlike stroma and immune escape by tumors that express the chemokine CCL21," Science, vol. 328, no. 5979, pp. 749-752, 2010.

[41] V. Mourmouras, M. Fimiani, P. Rubegni et al., "Evaluation of tumour-infiltrating CD4+CD25+FOXP3+ regulatory T cells in human cutaneous benign and atypical naevi, melanomas and melanoma metastases," British Journal of Dermatology, vol. 157, no. 3, pp. 531-539, 2007.

[42] R. Speeckaert, K. Vermaelen, N. van Geel et al., "Indoleamine2,3-dioxygenase, a new prognostic marker in sentinel lymph nodes of melanoma patients," European Journal of Cancer. In press.

[43] L. Vence, A. K. Palucka, J. W. Fay et al., "Circulating tumor antigen-specific regulatory $\mathrm{T}$ cells in patients with metastatic melanoma," Proceedings of the National Academy of Sciences 
of the United States of America, vol. 104, no. 52, pp. 20884 20889, 2007.

[44] T. O. Jensen, H. Schmidt, H. J. Moller et al., "Intratumoral neutrophils and plasmacytoid dendritic cells indicate poor prognosis and are associated with pSTAT3 expression in AJCC stage I/II melanoma," Cancer, vol. 118, no. 9, pp. 24762485, 2012.

[45] G. Gerlini, C. Urso, G. Mariotti et al., "Plasmacytoid dendritic cells represent a major dendritic cell subset in sentinel lymph nodes of melanoma patients and accumulate in metastatic nodes," Clinical Immunology, vol. 125, no. 2, pp. 184193, 2007.

[46] G. Gerlini, P. Di Gennaro, G. Mariotti et al., "Indoleamine 2,3-dioxygenase cells correspond to the BDCA2 plasmacytoid dendritic cells in human melanoma sentinel nodes," Journal of Investigative Dermatology, vol. 130, no. 3, pp. 898901, 2010.

[47] G. Konjević, K. Miräjaić Martinovi, V. Jurišić, N. Babović, and I. Spužić, "Biomarkers of suppressed natural killer (NK) cell function in metastatic melanoma: decreased NKG2D and increased CD158a receptors on CD3-CD16+ NK cells," Biomarkers, vol. 14, no. 4, pp. 258-270, 2009.

[48] K. Mirjacic Martinovic, G. Konjevic, N. Babovic, and M. Inic, "The stage dependent changes in NK cell activity and the expression of activating and inhibitory NK cell receptors in melanoma patients," Journal of Surgical Research, vol. 171, no. 2, pp. 637-649, 2011.

[49] A. Zippelius, P. Batard, V. Rubio-Godoy et al., "Effector function of human tumor-specific CD8 T cells in melanoma lesions: a state of local functional tolerance," Cancer Research, vol. 64, no. 8, pp. 2865-2873, 2004.

[50] C. A. Pepe, R. Ricci, C. Cortelazzi et al., "The vast majority of lymphocytes infiltrating primary cutaneous melanoma express the CD27 costimulatory receptor: implications for melanoma progression," European Journal of Dermatology, vol. 21, no. 2, pp. 178-183, 2011.

[51] M. R. Zubieta, D. Furman, M. Barrio, A. I. Bravo, E. Domenichini, and J. Mordoh, "Galectin-3 expression correlates with apoptosis of tumor-associated lymphocytes in human melanoma biopsies," American Journal of Pathology, vol. 168, no. 5, pp. 1666-1675, 2006.

[52] G. Radosavljevic, I. Jovanovic, I. Majstorovic et al., "Deletion of galectin-3 in the host attenuates metastasis of murine melanoma by modulating tumor adhesion and NK cell activity," Clinical and Experimental Metastasis, vol. 28, no. 5, pp. 451-462, 2011.

[53] S. I. Grivennikov, F. R. Greten, and M. Karin, "Immunity, inflammation, and cancer," Cell, vol. 140, no. 6, pp. 883-899, 2010.

[54] H. E. Kanavy and M. R. Gerstenblith, "Ultraviolet radiation and melanoma," Seminars in Cutaneous Medicine and Surgery, vol. 30, pp. 222-228, 2011.

[55] S. I. Grivennikov and M. Karin, "Dangerous liaisons: STAT3 and NF- $\kappa \mathrm{B}$ collaboration and crosstalk in cancer," Cytokine and Growth Factor Reviews, vol. 21, no. 1, pp. 11-19, 2010.

[56] A. Sica, P. Allavena, and A. Mantovani, "Cancer related inflammation: the macrophage connection," Cancer Letters, vol. 267, no. 2, pp. 204-215, 2008.

[57] S. Gazzaniga, A. I. Bravo, A. Guglielmotti et al., "Targeting tumor-associated macrophages and inhibition of MCP-1 reduce angiogenesis and tumor growth in a human melanoma xenograft," Journal of Investigative Dermatology, vol. 127, no. 8, pp. 2031-2041, 2007.
[58] P. Chen, Y. Huang, R. Bong et al., "Tumor-associated macrophages promote angiogenesis and melanoma growth via adrenomedullin in a paracrine and autocrine manner," Clinical Cancer Research, vol. 17, no. 23, pp. 7230-7239, 2011.

[59] F. Bianchini, D. Massi, C. Marconi et al., "Expression of cyclo-oxygenase- 2 in macrophages associated with cutaneous melanoma at different stages of progression," Prostaglandins and Other Lipid Mediators, vol. 83, no. 4, pp. 320-328, 2007.

[60] T. Fujimura, S. Ring, V. Umansky, K. Mahnke, and A. H. Enk, "Regulatory $\mathrm{T}$ cells stimulate B7-H1 expression in myeloid-derived suppressor cells in ret melanomas," Journal of Investigative Dermatology, vol. 132, no. 4, pp. 1239-1246, 2012.

[61] C. Meyer, A. Sevko, M. Ramacher et al., "Chronic inflammation promotes myeloid-derived suppressor cell activation blocking antitumor immunity in transgenic mouse melanoma model," Proceedings of the National Academy of Sciences of the United States of America, vol. 108, no. 41, pp. 1711117116, 2011.

[62] B. Toh, X. Wang, J. Keeble et al., "Mesenchymal transition and dissemination of cancer cells is driven by myeloidderived suppressor cells infiltrating the primary tumor," PLoS Biology, vol. 9, no. 9, Article ID e1001162, 2011.

[63] I. Poschke, D. Mougiakakos, J. Hansson, G. V. Masucci, and R. Kiessling, "Immature immunosuppressive CD14+HLADR-/low cells in melanoma patients are Stat3hi and overexpress CD80, CD83, and DC-sign," Cancer Research, vol. 70, no. 11, pp. 4335-4345, 2010.

[64] M. J. Slattery and C. Dong, "Neutrophils influence melanoma adhesion and migration under flow conditions," International Journal of Cancer, vol. 106, no. 5, pp. 713-722, 2003.

[65] H. Schmidt, S. Suciu, C. J. Punt et al., "Pretreatment levels of peripheral neutrophils and leukocytes as independent predictors of overall survival in patients with American Joint Committee on Cancer Stage IV Melanoma: results of the EORTC 18951 biochemotherapy trial," Journal of Clinical Oncology, vol. 25, no. 12, pp. 1562-1569, 2007.

[66] M. Artuc, S. Guhl, M. Babina et al., "Mast cell-derived TNF-alpha and histamine modify IL-6 and IL-8 expression and release from cutaneous tumor cells," Experimental Dermatology, vol. 20, pp. 1020-1022, 2011.

[67] R. Toth-Jakatics, S. Jimi, S. Takebayashi, and N. Kawamoto, "Cutaneous malignant melanoma: correlation between neovascularization and peritumor accumulation of mast cells overexpressing vascular endothelial growth factor," Human Pathology, vol. 31, no. 8, pp. 955-960, 2000.

[68] D. Ribatti, M. G. Ennas, A. Vacca et al., "Tumor vascularity and tryptase-positive mast cells correlate with a poor prognosis in melanoma," European Journal of Clinical Investigation, vol. 33, no. 5, pp. 420-425, 2003.

[69] J. M. Kirkwood, J. Manola, J. Ibrahim, V. Sondak, M. S. Ernstoff, and U. Rao, "A pooled analysis of Eastern cooperative oncology group and intergroup trials of adjuvant high-dose interferon for melanoma," Clinical Cancer Research, vol. 10, no. 5, pp. 1670-1677, 2004.

[70] A. A. Tarhini, J. M. Kirkwood, W. E. Gooding, C. Cai, and S. S. Agarwala, "Durable complete responses with high-dose bolus interleukin-2 in patients with metastatic melanoma who have experienced progression after biochemotherapy," Journal of Clinical Oncology, vol. 25, no. 25, pp. 3802-3807, 2007.

[71] M. J. Maeurer, S. M. Gollin, D. Martin et al., "Tumor escape from immune recognition: lethal recurrent melanoma in a patient associated with downregulation of the peptide 
transporter protein TAP-1 and loss of expression of the immunodominant MART-1/Melan-A antigen," The Journal of Clinical Investigation, vol. 98, no. 7, pp. 1633-1641, 1996.

[72] P. B. Chapman, A. Hauschild, C. Robert et al., "Improved survival with vemurafenib in melanoma with BRAF V600E mutation," The New England Journal of Medicine, vol. 364, no. 26, pp. 2507-2516, 2011.

[73] B. Comin-Anduix, T. Chodon, H. Sazegar et al., "The oncogenic BRAF kinase inhibitor PLX4032/RG7204 does not affect the viability or function of human lymphocytes across a wide range of concentrations," Clinical Cancer Research, vol. 16, no. 24, pp. 6040-6048, 2010.

[74] J. S. Wilmott, G. V. Long, J. R. Howle et al., "Selective BRAF inhibitors induce marked $\mathrm{T}$ cell infiltration into human metastatic melanoma," Clinical Cancer Research, vol. 18, no. 5, pp. 1386-1394, 2012.

[75] K. Rakhra, P. Bachireddy, T. Zabuawala et al., "CD4+ T cells contribute to the remodeling of the microenvironment required for sustained tumor regression upon oncogene inactivation," Cancer Cell, vol. 18, no. 5, pp. 485-498, 2010.

[76] H. T. Khong and S. A. Rosenberg, "Pre-existing immunity to tyrosinase-related protein (TRP)-2, a new TRP-2 isoform, and the NY-ESO-1 melanoma antigen in a patient with a dramatic response to immunotherapy," Journal of Immunology, vol. 168, no. 2, pp. 951-956, 2002.

[77] C. Germeau, W. Ma, F. Schiavetti et al., "High frequency of antitumor $\mathrm{T}$ cells in the blood of melanoma patients before and after vaccination with tumor antigens," Journal of Experimental Medicine, vol. 201, no. 2, pp. 241-248, 2005.

[78] C. Lurquin, B. Lethé, E. De Plaen et al., "Contrasting frequencies of antitumor and anti-vaccine T cells in metastases of a melanoma patient vaccinated with a MAGE tumor antigen," Journal of Experimental Medicine, vol. 201, no. 2, pp. 249$257,2005$.

[79] A. Bins, H. Mallo, J. Sein et al., "Phase I clinical study with multiple peptide vaccines in combination with tetanus toxoid and GM-CSF in advanced-stage HLA-A*0201-positive melanoma patients," Journal of Immunotherapy, vol. 30, no. 2, pp. 234-239, 2007.

[80] F. S. Hodi, S. J. O’Day, D. F. McDermott et al., "Improved survival with ipilimumab in patients with metastatic melanoma," The New England Journal of Medicine, vol. 363, no. 8, pp. 711-723, 2010.

[81] K. Klages, C. T. Mayer, K. Lahl et al., "Selective depletion of Foxp3+ regulatory $\mathrm{T}$ cells improves effective therapeutic vaccination against established melanoma," Cancer Research, vol. 70, no. 20, pp. 7788-7799, 2010.

[82] M. M. Barrio, P. T. de Motta, J. Kaplan et al., "A phase I study of an allogeneic cell vaccine (VACCIMEL) with GM-CSF in melanoma patients," Journal of Immunotherapy, vol. 29, no. 4, pp. 444-454, 2006.

[83] E. M. von Euw, M. M. Barrio, D. Furman et al., "Monocytederived dendritic cells loaded with a mixture of apoptotic/ necrotic melanoma cells efficiently cross-present gp100 and MART-1 antigens to specific CD8+ T lymphocytes," Journal of Translational Medicine, vol. 5, article 19, 2007.

[84] E. M. von Euw, M. M. Barrio, D. Furman et al., "A phase I clinical study of vaccination of melanoma patients with dendritic cells loaded with allogeneic apoptotic/necrotic melanoma cells. Analysis of toxicity and immune response to the vaccine and of IL-10-1082 promoter genotype as predictor of disease progression," Journal of Translational Medicine, vol. 6, article 6, 2008.
[85] L. Hershkovitz, J. Schachter, A. J. Treves, and M. J. Besser, "Focus on adoptive T cell transfer trials in melanoma," Clinical and Developmental Immunology, vol. 2010, Article ID 260267, 11 pages, 2010.

[86] M. E. Dudley, J. R. Wunderlich, J. C. Yang et al., "Adoptive cell transfer therapy following non-myeloablative but lymphodepleting chemotherapy for the treatment of patients with refractory metastatic melanoma," Journal of Clinical Oncology, vol. 23, no. 10, pp. 2346-2357, 2005.

[87] M. E. Dudley, J. R. Wunderlich, P. F. Robbins et al., "Cancer regression and autoimmunity in patients after clonal repopulation with antitumor lymphocytes," Science, vol. 298, no. 5594, pp. 850-854, 2002.

[88] M. E. Dudley, J. C. Yang, R. Sherry et al., "Adoptive cell therapy for patients with metastatic melanoma: evaluation of intensive myeloablative chemoradiation preparative regimens," Journal of Clinical Oncology, vol. 26, no. 32, pp. 52335239, 2008

[89] M. J. Besser, R. Shapira-Frommer, A. J. Treves et al., "Clinical responses in a phase II study using adoptive transfer of shortterm cultured tumor infiltration lymphocytes in metastatic melanoma patients," Clinical Cancer Research, vol. 16, no. 9, pp. 2646-2655, 2010.

[90] R. A. Morgan, M. E. Dudley, J. R. Wunderlich et al., "Cancer regression in patients after transfer of genetically engineered lymphocytes," Science, vol. 314, no. 5796, pp. 126-129, 2006.

[91] L. A. Johnson, R. A. Morgan, M. E. Dudley et al., "Gene therapy with human and mouse $\mathrm{T}$-cell receptors mediates cancer regression and targets normal tissues expressing cognate antigen," Blood, vol. 114, no. 3, pp. 535-546, 2009.

[92] C. Yee, J. A. Thompson, D. Byrd et al., "Adoptive T cell therapy using antigen-specific CD8+ $\mathrm{T}$ cell clones for the treatment of patients with metastatic melanoma: in vivo persistence, migration, and antitumor effect of transferred $\mathrm{T}$ cells," Proceedings of the National Academy of Sciences of the United States of America, vol. 99, no. 25, pp. 16168-16173, 2002.

[93] A. Mackensen, N. Meidenbauer, S. Vogl, M. Laumer, J. Berger, and R. Andreesen, "Phase I study of adoptive T-cell therapy using antigen-specific CD8+ T cells for the treatment of patients with metastatic melanoma," Journal of Clinical Oncology, vol. 24, no. 31, pp. 5060-5069, 2006.

[94] T. Reya, S. J. Morrison, M. F. Clarke, and I. L. Weissman, "Stem cells, cancer, and cancer stem cells," Nature, vol. 414, no. 6859 , pp. 105-111, 2001.

[95] E. Quintana, M. Shackleton, H. R. Foster et al., "Phenotypic heterogeneity among tumorigenic melanoma cells from patients that is reversible and not hierarchically organized," Cancer Cell, vol. 18, no. 5, pp. 510-523, 2010.

[96] A. D. Boiko, O. V. Razorenova, M. van de Rijn et al., "Human melanoma-initiating cells express neural crest nerve growth factor receptor CD271," Nature, vol. 466, pp. 133-137, 2010.

[97] T. Schatton, U. Schütte, N. Y. Frank et al., "Modulation of T-cell activation by malignant melanoma initiating cells," Cancer Research, vol. 70, no. 2, pp. 697-708, 2010.

[98] E. Quintana, M. Shackleton, M. S. Sabel, D. R. Fullen, T. M. Johnson, and S. J. Morrison, "Efficient tumour formation by single human melanoma cells," Nature, vol. 456, no. 7222, pp. 593-598, 2008.

[99] P. F. Robbins, M. E. Dudley, J. Wunderlich et al., "Cutting edge: persistence of transferred lymphocyte clonotypes correlates with cancer regression in patients receiving cell transfer therapy," Journal of Immunology, vol. 173, no. 12, pp. 7125-7130, 2004. 
[100] S. A. Rosenberg and M. E. Dudley, "Cancer regression in patients with metastatic melanoma after the transfer of autologous antitumor lymphocytes," Proceedings of the National Academy of Sciences of the United States of America, vol. 101, supplement 2, pp. 14639-14645, 2004.

[101] M. Aris, M. R. Zubieta, M. Colombo et al., "MART-1- and gp100-expressing and -non-expressing melanoma cells are equally proliferative in tumors and clonogenic in vitro," Journal of Investigative Dermatology, vol. 132, pp. 365-374, 2012.

[102] F. Lozupone, L. Rivoltini, F. Luciani et al., "Adoptive transfer of an anti-MART-127-35-specific CD8+ T cell clone leads to immunoselection of human melanoma antigen-loss variants in SCID mice," European Journal of Immunology, vol. 33, no. 2, pp. 556-566, 2003.

[103] L. Sanchez-Perez, T. Kottke, R. M. Diaz et al., "Potent selection of antigen loss variants of B16 melanoma following inflammatory killing of melanocytes in vivo," Cancer Research, vol. 65, no. 5, pp. 2009-2017, 2005.

[104] L. Paco, A. M. Garcia-Lora, C. Casares et al., "Total loss of HLA class I expression on a melanoma cell line after growth in nude mice in absence of autologous antitumor immune response," International Journal of Cancer, vol. 121, no. 9, pp. 2023-2030, 2007.

[105] C. Garrido, I. Algarra, I. Maleno et al., "Alterations of HLA class i expression in human melanoma xenografts in immunodeficient mice occur frequently and are associated with higher tumorigenicity," Cancer Immunology, Immunotherapy, vol. 59, no. 1, pp. 13-26, 2010.

[106] F. Lehmann, M. Marchand, P. Hainaut et al., "Differences in the antigens recognized by cytolytic $\mathrm{T}$ cells on two successive metastases of a melanoma patient are consistent with immune selection," European Journal of Immunology, vol. 25, no. 2, pp. 340-347, 1995.

[107] E. Jäger, M. Ringhoffer, J. Karbach, M. Arand, F. Oesch, and A. Knuth, "Inverse relationship of melanocyte differentiation antigen expression in melanoma tissues and CD8+ cytotoxicT-cell responses: evidence for immunoselection of antigenloss variants in vivo," International Journal of Cancer, vol. 66, no. 4, pp. 470-476, 1996.

[108] N. P. Restifo, F. M. Marincola, Y. Kawakami, J. Taubenberger, J. R. Yannelli, and S. A. Rosenberg, "Loss of functional beta2microglobulin in metastatic melanomas from five patients receiving immunotherapy," Journal of the National Cancer Institute, vol. 88, no. 2, pp. 100-108, 1996.

[109] E. Jager, M. Ringhoffer, M. Altmannsberger et al., "Immunoselection in vivo: independent loss of MHC class I and melanocyte differentiation antigen expression in metastatic melanoma," International Journal of Cancer, vol. 71, no. 2, pp. 142-147, 1997.

[110] H. T. Khong, Q. J. Wang, and S. A. Rosenberg, "Identification of multiple antigens recognized by tumor-infiltrating lymphocytes from a single patient: tumor escape by antigen loss and loss of MHC expression," Journal of Immunotherapy, vol. 27, no. 3, pp. 184-190, 2004.

[111] B. Riteau, F. Faure, C. Menier et al., "Exosomes bearing HLA$\mathrm{G}$ are released by melanoma cells," Human Immunology, vol. 64, no. 11, pp. 1064-1072, 2003.

[112] M. B. Fuertes, M. V. Girart, L. L. Molinero et al., "Intracellular retention of the NKG2D ligand MHC class i chain-related gene a in human melanomas confers immune privilege and prevents NK cell-mediated cytotoxicity," Journal of Immunology, vol. 180, no. 7, pp. 4606-4614, 2008.
[113] L. Derré, M. Corvaisier, B. Charreau et al., "Expression and release of HLA-E by melanoma cells and melanocytes: potential impact on the response of cytotoxic effector cells," Journal of Immunology, vol. 177, no. 5, pp. 3100-3107, 2006.

[114] J. R. Brody, C. L. Costantino, A. C. Berger et al., "Expression of indoleamine 2,3-dioxygenase in metastatic malignant melanoma recruits regulatory $\mathrm{T}$ cells to avoid immune detection and affects survival," Cell Cycle, vol. 8, no. 12, pp. 1930-1934, 2009.

[115] G. C. Prendergast, R. Metz, and A. J. Muller, "IDO recruits tregs in melanoma," Cell Cycle, vol. 8, no. 12, pp. 1818-1819, 2009.

[116] N. Martin-Orozco, Y. Li, Y. Wang et al., "Melanoma cells express ICOS ligand to promote the activation and expansion of T-regulatory cells," Cancer Research, vol. 70, no. 23, pp. 9581-9590, 2010.

[117] E. Itakura, R. R. Huang, D. R. Wen, E. Paul, P. H. Wünsch, and A. J. Cochran, "IL-10 expression by primary tumor cells correlates with melanoma progression from radial to vertical growth phase and development of metastatic competence," Modern Pathology, vol. 24, no. 6, pp. 801-809, 2011.

[118] N. Schoof, M. M. Iles, D. T. Bishop, J. A. Newton-Bishop, J. H. Barrett, and GenoMEL Consortium, "Pathway-based analysis of a melanoma genome-wide association study: analysis of genes related to tumour-immunosuppression," PLOS ONE, vol. 6, no. 12, Article ID e29451, 2011.

[119] M. Hahne, D. Rimoldi, M. Schröter et al., "Melanoma cell expression of Fas(Apo-1/CD95) ligand: implications for tumor immune escape," Science, vol. 274, no. 5291, pp. 13631366, 1996.

[120] H. Dong, S. E. Strome, D. R. Salomao et al., "Tumorassociated $\mathrm{B} 7-\mathrm{H} 1$ promotes T-cell apoptosis: a potential mechanism of immune evasion," Nature Medicine, vol. 8, article 1039, pp. 793-800, 2002.

[121] G. Andreola, L. Rivoltini, C. Castelli et al., "Induction of lymphocyte apoptosis by tumor cell secretion of FasLbearing microvesicles," Journal of Experimental Medicine, vol. 195, no. 10, pp. 1303-1316, 2002.

[122] M. J. Martínez-Lorenzo, A. Anel, M. A. Alava et al., “The human melanoma cell line MelJuSo secretes bioactive FasL and APO2L/TRAIL on the surface of microvesicles. Possible contribution to tumor counterattack," Experimental Cell Research, vol. 295, no. 2, pp. 315-329, 2004.

[123] E. R. Brown, T. Doig, N. Anderson et al., "Association of galectin-3 expression with melanoma progression and prognosis," European Journal of Cancer, vol. 48, no. 6, pp. 865874, 2012.

[124] E. Lesport, J. Baudhuin, J. LeMaoult et al., "Human melanoma cell secreting human leukocyte antigen-G5 inhibit natural killer cell cytotoxicity by impairing lytic granules polarization toward target cell," Human Immunology, vol. 70, no. 12, pp. 1000-1005, 2009.

[125] G. Konjevic, K. Mirjacic Martinovic, A. Vuletic et al., "Low expression of CD161 and NKG2D activating NK receptor is associated with impaired NK cell cytotoxicity in metastatic melanoma patients," Clinical and Experimental Metastasis, vol. 24, no. 1, pp. 1-11, 2007.

[126] V. Umansky and A. Sevko, "Overcoming immunosuppression in the melanoma microenvironment induced by chronic inflammation," Cancer Immunology, Immunotherapy, vol. 61, no. 2, pp. 275-282, 2012.

[127] Y. Guilloux, C. Viret, N. Gervois et al., "Defective lymphokine production by most $\mathrm{CD} 8+$ and $\mathrm{CD} 4+$ tumor-specific $\mathrm{T}$ 
cell clones derived from human melanoma-infiltrating lymphocytes in response to autologous tumor cells in vitro," European Journal of Immunology, vol. 24, no. 9, pp. 19661973, 1994.

[128] A. K. Simon, E. Jones, H. Richards et al., "Regulatory T cells inhibit Fas ligand-induced innate and adaptive tumour immunity," European Journal of Immunology, vol. 37, no. 3, pp. 758-767, 2007.

[129] M. Viguier, F. Lemaître, O. Verola et al., "Foxp3 expressing $\mathrm{CD} 4+\mathrm{CD} 25$ high regulatory $\mathrm{T}$ cells are overrepresented in human metastatic melanoma lymph nodes and inhibit the function of infiltrating T cells," Journal of Immunology, vol. 173, no. 2, pp. 1444-1453, 2004.

[130] M. D. McCarter, J. Baumgartner, G. A. Escobar et al., "Immunosuppressive dendritic and regulatory $\mathrm{T}$ cells are upregulated in melanoma patients," Annals of Surgical Oncology, vol. 14, no. 10, pp. 2854-2860, 2007.

[131] K. Tsuji, T. Hamada, A. Uenaka et al., "Induction of immune response against NY-ESO-1 by CHP-NY-ESO-1 vaccination and immune regulation in a melanoma patient," Cancer Immunology, Immunotherapy, vol. 57, no. 10, pp. 1429-1437, 2008.

[132] T. Nicholaou, L. M. Ebert, I. D. Davis et al., "Regulatory Tcell-mediated attenuation of T-cell responses to the NY-ESO1ISCOMATRIX vaccine in patients with advanced malignant melanoma," Clinical Cancer Research, vol. 15, no. 6, pp. 21662173, 2009.

[133] F. Zhao, C. Falk, W. Osen, M. Kato, D. Schadendorf, and V. Umansky, "Activation of p38 mitogen-activated protein kinase drives dendritic cells to become tolerogenic in ret transgenic mice spontaneously developing melanoma," Clinical Cancer Research, vol. 15, no. 13, pp. 4382-4390, 2009.

[134] D. H. Munn, M. D. Sharma, D. Hou et al., "Expression of indoleamine 2,3-dioxygenase by plasmacytoid dendritic cells in tumor-draining lymph nodes," The Journal of Clinical Investigation, vol. 114, no. 4, p. 599, 2004.

[135] J. Charles, J. Di Domizio, D. Salameire et al., "Characterization of circulating dendritic cells in melanoma: role of CCR6 in plasmacytoid dendritic cell recruitment to the tumor," Journal of Investigative Dermatology, vol. 130, no. 6, pp. 16461656, 2010. 


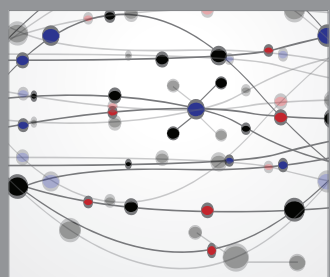

The Scientific World Journal
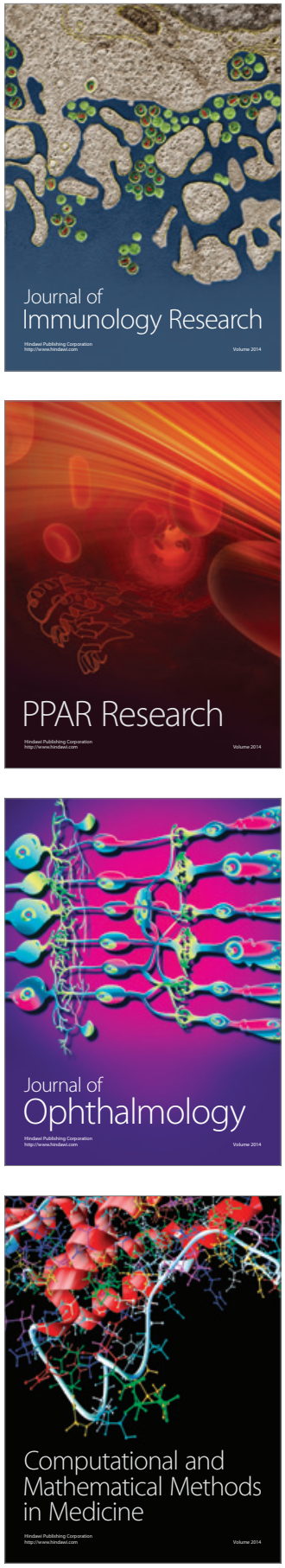

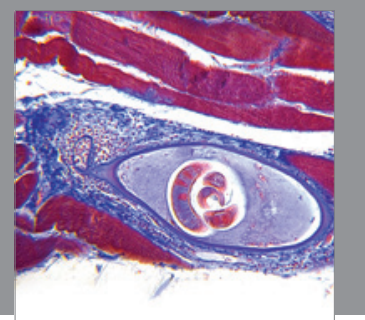

Gastroenterology

Research and Practice
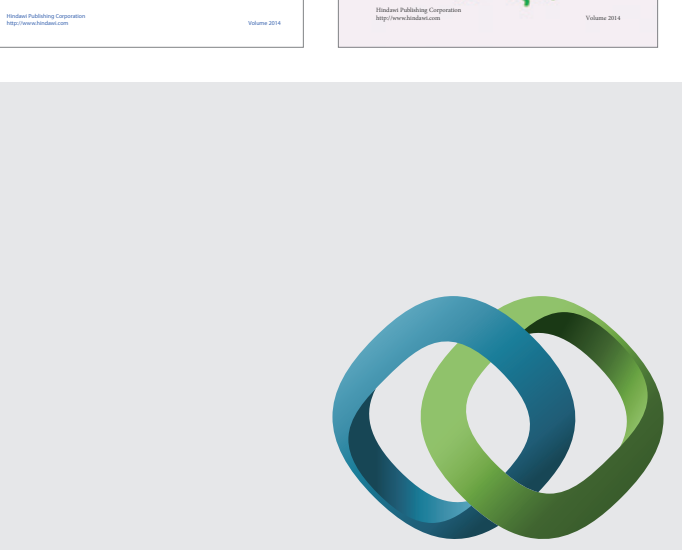

\section{Hindawi}

Submit your manuscripts at

http://www.hindawi.com
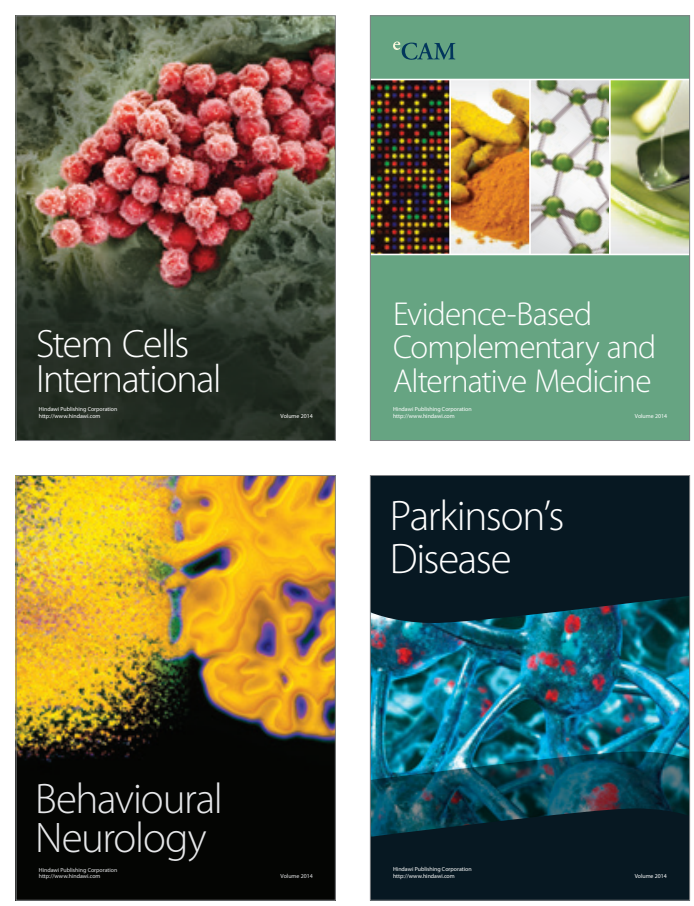

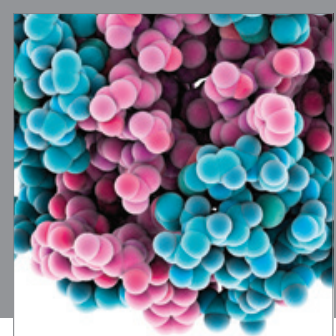

Journal of
Diabetes Research

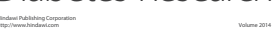

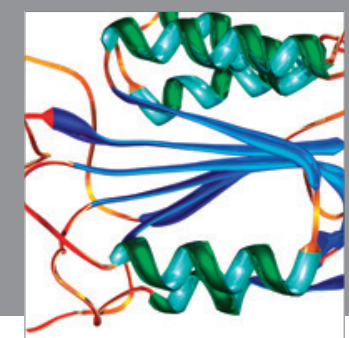

Disease Markers
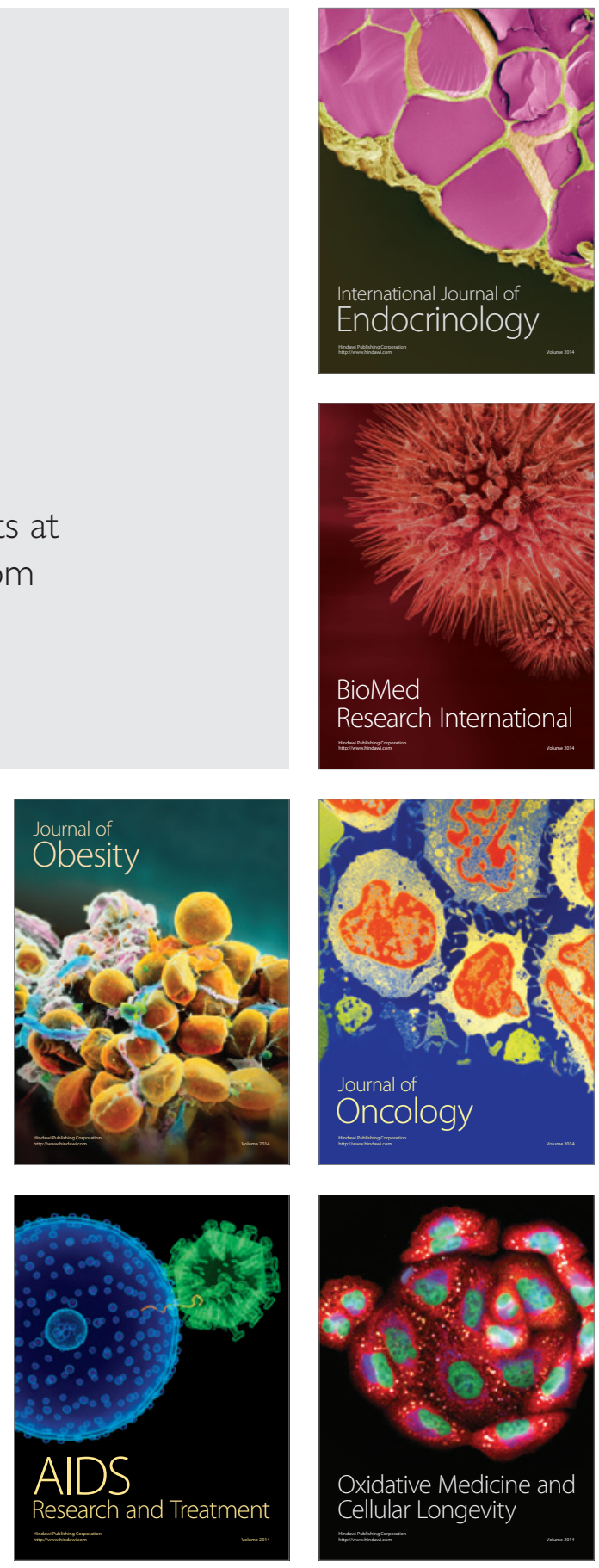